\title{
Granulomatosis with polyangitis (WEGENER'S)- Strawberry gingivitis as prime manifestation
}

\author{
Jesmith Elsa Jacob ${ }^{1 *}$, Asish Rajasekharan ${ }^{2}$, Tinky Bose $\mathrm{C}^{3}$, Ramesh $\mathrm{S}^{4}$, Vinimol $\mathrm{C}^{5}$, Akhilanand Chaurasia ${ }^{6}$

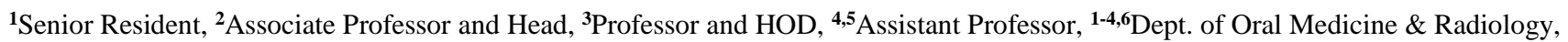 \\ ${ }^{5}$ Dept. of Pharmacology, ${ }^{\mathbf{1 , 2 , 4}}$ Government Dental College, Alappuzha, Kerala, ${ }^{\mathbf{3}}$ Government Dental College, Thiruvananthapuram, Kerala, \\ ${ }^{5}$ Government Medical College, Kollam, Kerala, ${ }^{6}$ King George Medical University, Lucknow, Uttar Pradesh, India
}

*Corresponding Author: Jesmith Elsa Jacob

Email: jesmithjacob7@gmail.com

\begin{abstract}
Wegener's granulomatosis (WG) is an immunologically mediated inflammatory disease. Major histological features include a necrotizing vasculitis involving small vessels, extensive "geographic" necrosis, and granulomatous inflammation. Clinical manifestations of WG are protean; virtually any organ can be involved. Further, the spectrum and severity of the disease is heterogeneous, ranging from indolent disease involving only one site to fulminant, multiorgan vasculitis leading to death. This case report documenrts the importance of considering Wegner's granulomatosis in patients with unique gingival lesions. The most common presenttion is a friable-granular-hyperplastic gingivitis associated with alveolar resorption and tooth mobility. The disease may remain localized to the mouth for several weeks or months before multiorgan involvement occurs. Gingival enlargement is a direct manifestation of Wegener granulomatosis, and it may be pathognomonic. Failure to recognize the clinical lesions can result in delayed diagnosis and treatment, with potentially fatal results. Therefore, the aim of reporting this case was to emphasize that, the dental surgeon often being the first person to examine the oral cavity, should be familiar with the typical appearance of gingival WG as "strawberry gingivitis," its clinical course as well as diagnostic parameters and adequate management.
\end{abstract}

Keywords: Strawberry gingivitis, Wegener's granulomatosis, Antineutrophil cytoplasmic bodies, Geographic necrosis.

\section{Introduction}

Wegener's granulomatosis (WG) is an immunologically mediated uncommon multisystem disorder, first described by Klinger in 1933 followed by other investigators, including Rossle in 1933, Friedrich Wegener in 1936. ${ }^{1}$ However, detailed description of WG is given by Godman and Churg. While WG typically affects the upper and lower airways, and frequently the kidneys, it may involve any organ system. The granulomatous inflammation and vasculitis can affect the mouth, eyes, ear, nose, throat, lungs, skin and kidneys. The disease predominantly affects adults with mean age of 41 years, but there is no significant gender predilection. In 1990, the American College of Rheumatology (ACR) proposed the following four specific criteria for the classification of $\mathrm{WG}$ : (1) oral ulcers or nasal discharge, (2) the presence of nodules, fixed infiltrates or cavities on a chest radiograph, (3) abnormal urinary sediment (red blood cell casts or more than five red blood cells per high power field) and (4) granulomatous inflammation on biopsy. For the diagnosis of WG, a minimum of two criteria should be fulfilled from the above-mentioned (ACR 1990) criteria. $^{2}$ As the dental surgeon is often the first person to examine the oral cavity, he should be familiar with the classical appearance of gingival WG as "strawberry gingivitis," its clinical course as well as diagnostic parameters and adequate management.

\section{Case Report}

A 31 year old female patient was reported with chief complaint of swelling and bleeding from front gum region of three weeks duration. Patient had noticed a small swelling in relation to upper front teeth around three weeks back. The swelling then rapidly increased and reached to the present size and had noticed similar swelling on lower front gum region in a short while. Swelling was associated with mild intermittent gnawing pain and bleeding from the front gum region. Pain and bleeding aggravates on having food and while brushing. Patient has not taken any medication for the same. She denied sinusitis, difficulty breathing, skin lesions or other systemic concerns. Her past medical history and general examination were unremarkable.

On intraoral examination, the maxillary and mandibular anterior quadrants of gingiva appears hyperplastic and reddish-purple in colour. The surface exhibits numerous short bulbous projections, which are hemorrhagic and friable and red bumpy surface resembles strawberry like appearance. (Fig. 1) The lesion is seen to extend from 16 to 25 with diffuse involvement of maxillary gingiva, partially covering the crowns of the teeth and is localized to interdental mandibular gingiva with lateral spread to adjacent areas. Non tender, friable and soft in consistency with profuse bleeding on palpation and probing were noted. Mandibular central and lateral incisors exhibited grade II mobility. A provisional diagnosis of orofacial granulomatosis was considered.

On routine blood examination, hemoglobin was $8.5 \%$. Evaluation of serum cytoplasmic anti neutrophilic cytoplasmic antibody (cANCA) was done, and it was increased to $39.2 \mathrm{mg} / \mathrm{dl}$, C-Reactive protein was also increased to $3.5 \mathrm{mg} / \mathrm{dl}$. Peripheral blood smear showed neutrophilia, hyperglobulinemia and leuckocytosis.

Panoramic radiograph did not reveal any significant finding except for the generalized horizontal bone loss with evident crestal bone loss in the mandibular anterior region (Fig. 2) 
An incisional biopsy of the gingiva in relation to 45 was performed under local anesthesia. On microscopic examination the haematoxylin and eosin stained sections showed an ulcerated stratified squamous epithelium overlying a connective tissue stroma showing granulomatous areas with epithelioid cells and intense collections of neutrophils, macrophages and plasma cells. Localized areas of necrosis and few multinucleated giant cells were also noticed. However necrotizing vasculitis was not evident. Special stains were negative for fungi and mycobacterial bacilli and no foreign material was evident. Cd1a immunostaining was performed to rule out the possibility of
Langerhans cell histiocytosis and proved to be negative. (Fig. 3)

Based on the history, the intraoral examination, and the radiographic data, the differential diagnoses included lupus erythematosus, leukemia, hypertrophic response to Candida or other organisms, pemphigus foliaceous, and Wegener granulomatosis. The patient has been started on Prednisolone $20 \mathrm{mg} /$ day on divided and tapering dose for 1 week with morning doses of $10 \mathrm{mg}$ after proper systemic evaluation. To give symptomatic relief, topical steroid as well astringing agents was also advised.
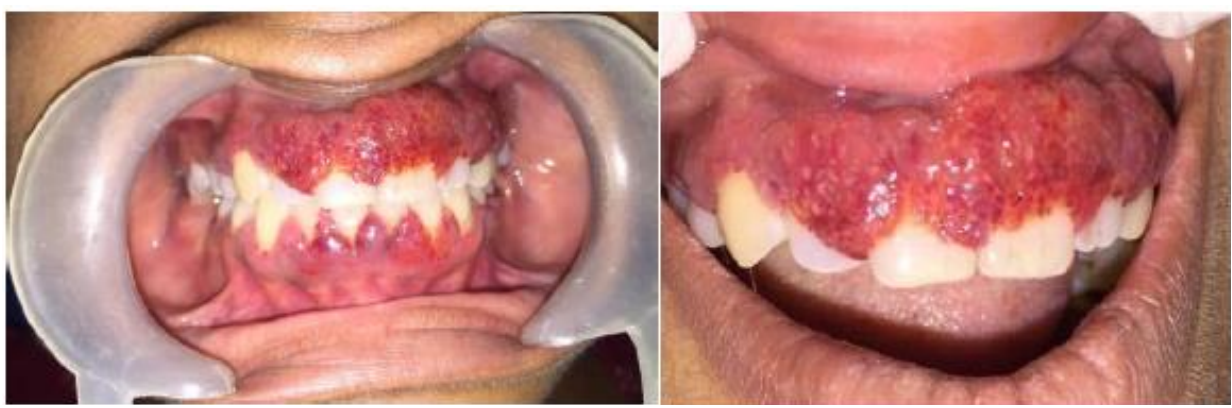

Fig. 1: Hemorrhagic and friable gingiva (Strawberry gingivitis)

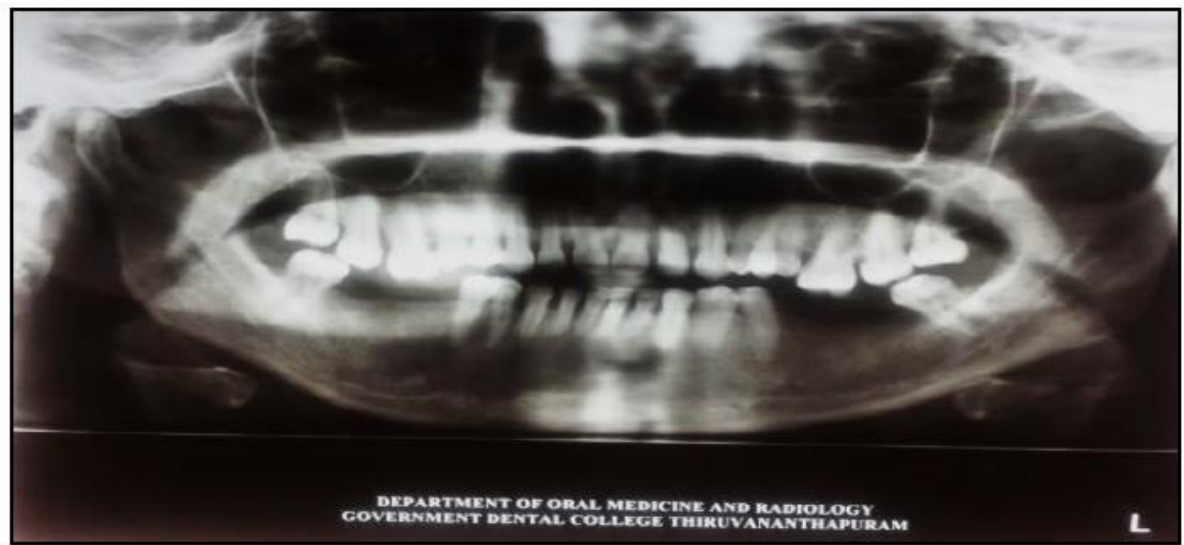

Fig. 2: Panoramic radiograph

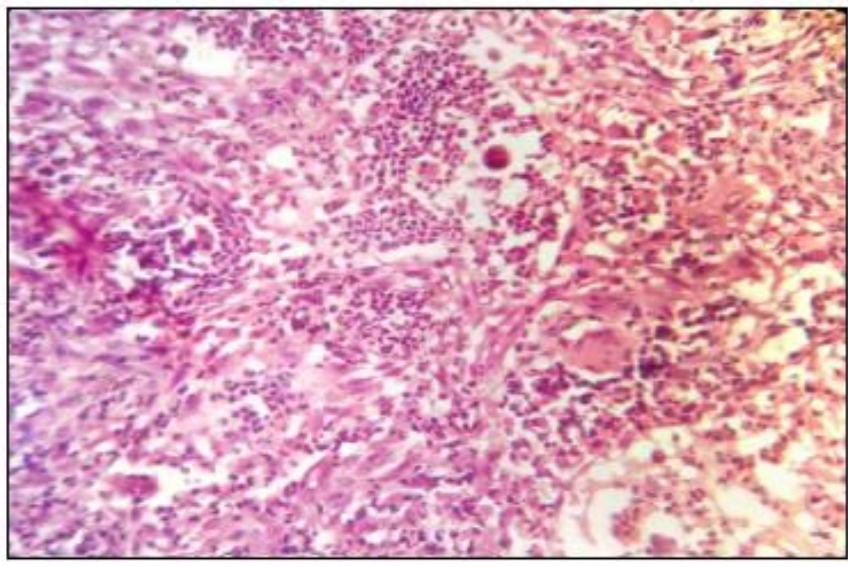

Fig. 3: Photomicrograph showing granulomatous areas with epitheloid cells, inflammatory cells and multinucleated giant cell $(\mathrm{H} / \mathrm{E}, 40 \mathrm{x}$ 


\section{Discussion}

The term "Wegener's granulomatosis was proposed to honor Dr. Friedrich Wegener. As more has been learned about the disease, it has become clear that it is a highly complex entity with diverse symptoms, signs, and outcomes, and the name "Wegener's granulomatosis" does not adequately represent an understanding of the disease. The new terminology for "Wegener's granulomatosis" which has been adopted by the American College of Rheumatology, the American Society of Nephrology, and the European League Against Rheumatism is "granulomatosis with polyangiitis (Wegener's)", which can be abbreviated as "GPA". The reasons for the change in terminology are outlined in a short manuscript recently published simultaneously in the journals of these three organizations (Arthritis and Rheumatism, Annals of the Rheumatic Diseases, and The Journal of the American Society of Nephrology). ${ }^{3}$

This case exemplifies the contribution dentists can make in the early recognition and treatment of Wegener granulomatosis. The dentist's contribution is especially important as untreated patients have a mean survival of 5 months, and treated patients show sustained disease remission.

WG is characterized as one of the C -ANCA associated small cell vasculitis which predominantly affects adults with mean age of 41 years. However no significant gender predilection has been reported. It is uncommon with a reported incidence of three per 100,000. Depending on the extent of systemic involvement, WG can be: a) classic, i.e., involving the upper and lower respiratory tract, b) generalized, where rapid involvement of the renal system occurs in untreated patients, c) limited, affecting the respiratory system without occurrence of renal lesions, and d) superficial, primarily exhibiting skin and mucosal lesions. When the classic triad of necrotizing granulomatous lesions of the respiratory tract, generalized vasculitis and necrotizing glomerulonephritis are absent, the diagnosis of WG is challenging. Occurrence of oral mucosal and cutaneous manifestations are invaluable in the diagnosis of WG. Only $6 \%$ of the cases were presented with oral manifestation, which were in advanced stage of the disease and are rarely an indicator of the disease.

After correlation of all positive findings Wegener's granulomatosis was diagnosed in our case on the basis of three key features: (1) the multiple gingival lesions, which resembled over-ripe strawberries; (2) the patient's complaint of malaise (supported by increased ESR and C-reactive protein concentration); and (3) the histopathological appearances in the gingival biopsy specimen. Otorhinolaryngological involvement is more common with destruction of nasal septum, resulting in palatal perforation and progressive sensorineural hearing loss. Ophthalmic symptoms may manifest as epiphora due to involvement of the nasolacrimal duct. Lesion of the skin manifests as purpura, nodules and ulcers, and has been reported in about $46 \%$ of the affected patients and in $13 \%$ of the affected patients at the onset of disease. However, limited forms of the disease have been reported, in which only one or two organ systems have been involved. ${ }^{4}$

This particular combination of clinical and histological features was first described in a peculiar form of progressive hyperplastic gingivitis by Milner, when the association with Wegener's granulomatosis was made only at necropsy. Small numbers of similar cases have been reported since, most of which draw attention to the delay between initial presentation and diagnosis. Although the histological picture of gingival biopsies often do not show vasculitis, important diagnostic features can be realized from these oral lesions, such as epithelial hyperplasia, micro abscesses, mixed inflammation, and scattered giant cells. ${ }^{5}$ The oral lesions may manifest either as mucosal ulcer on the tongue, buccal mucosa, gums and palate, or as gingival hyperplasia with classical "strawberry gingivitis." However, Cawson suggested that other lesions may also occur, such as ulceration of the palate by extension from the nose, where destruction of the nasal septum may develop. It may also occur as small ulcer-like aphthae, diffuse ulcerative stomatitis and spontaneous exfoliation of the teeth, as seen in our case.

The clinical differential diagnosis might include: leukemia, drug-induced gingival hyperplasia, exuberant infection, and pregnancy gingivitis. These disorders can be excluded by medical and dental history, by examination, and by laboratory studies. ${ }^{6}$

In the case presented, diagnosis of WG was made according to the criteria given by the American Association of Rheumatology, after careful exclusion of the abovementioned lesions by appropriate systemic evaluation, lab investigation and referring the previous reported case with similar clinical and histopathological reports. Our case also fulfilled two criteria of the ACR 1990, including gingival ulceration, which appeared like "strawberry gingivitis," and granulomatous lesion in gingival biopsy.

Although the exact cause of Wegener granulomatosis is unknown, it is considered to be a hypersensitivity response. Granulomatous vasculitis and glomerulonephritis are characteristic of hypersensitivity states.15 No offending antigen or noxious agent has been isolated.

Most authors acknowledge that the clinicopathological complex of "strawberry gums" and the accompanying histopathological features of pseudoepitheliomatous hyperplasia, intraepithelial abscess penetrating the surface epithelium, microabscesses in connective tissue stroma and multinucleate giant cells are "highly suggestive" of WG. It was also suggested that there was no association of the features of this complex with any other disease process and, in an appropriate clinical setting, they are so characteristic of gingival WG as to be virtually diagnostic, particularly as the classic criteria of vasculitis, granulomata and necrosis occur only rarely in gingival biopsy specimens. The diagnosis of WG was made in the case presented based on these particular combination of clinical and histopathologic findings. ${ }^{7}$

The serological tests helped only to reinforce the clinical impression of a systemic inflammatory process and did not confirm the diagnosis of WG. The cANCA titer, arguably the most useful investigation in cases of WG, was contributory 
in our case. Even the presence of the nasal lesion could not be considered diagnostic. ${ }^{8}$

The corticosteroid treatment was regarded as an interim measure pending final diagnosis. Cytotoxic agents, particularly cyclophosphamide, are favored in the disease since cyclophosphamide can promote healing and even total remission. The treatment regimen in this case included glucocorticosteroids (up to $1 \mathrm{mg}$ per $\mathrm{kg}$ per day) in conjunction with cyclophosphamide (1 to $2 \mathrm{mg}$ per $\mathrm{kg}$ per day). When possible, the steroid therapy is tapered to an alternate-day schedule, and both drugs are tapered after 1 year of treatment. Currently, short-course (3-6 months) induction treatment with CYC plus CS, followed by maintenance therapy with less toxic agents (e.g., methotrexate, azathioprine) is recommended. Further, recent studies suggest that methotrexate combined with CS may be adequate for limited, non-life threatening WG. The role of other immunomodulatory agents (including trimethoprimsulfamethoxazole) is also explored. Good prognosis with long-term remissions has been reported with combination therapy. The reported case responded to steroid therapy. ${ }^{10}$

If left untreated, WG is often fatal within the first year of onset. Mean survival is reported as 5 years for untreated cases. The condition has a more favorable prognosis in the absence of renal involvement.

\section{Conclusion}

The characteristic gingival lesion with the combination of histopathology might be diagnostic of WG, and early diagnosis and aggressive treatment is of paramount importance to achieve optimum treatment outcomes. Awareness of the wide spectrum of clinical presentations, differentiating features and clinical course of WG is essential for prompt diagnosis and appropriate management.

The picture of exuberant gingiva, granular friable tissue, and scattered petechial areas is unique, and it is valuable in the early diagnosis of Wegener granulomatosis. As no other disease is known to produce this characteristic picture, gingivitis should be considered pathognomonic of Wegener granulomatosis, and should prompt dentists to refer patients immediately for medical evaluation and treatment. ${ }^{11}$

\section{Source of funding}

There was no external source of funding for the study.

\section{Conflicts of interest}

The authors report no conflicts of interest related to this study.

\section{References}

1. F. W. Uber generalisierte, septische Gefaesserkrankungen. Verh Dtsch Ges Pathol 1936;29:202-10.

2. Manchanda Y, Tejasvi T, Handa R RM. Strawberry gingiva: a distinctive sign in Wegener's granulomatosis. $J$ Am Acad Dermatol 2003;49:335-7.

3. Lynch III JP TH. Wegener granulomatosis (granulomatosis with polyangiitis): evolving concepts in treatment. Semin Respir Crit Care Med 2011;32(3):274-97.

4. Heera R, Choudhary K, Beena VT SR. Strawberry gingivitis: a diagnostic feature of gingival Wegener's granulomatosis! Dent Res J 2012;9(S):123-6.

5. Cohen PS MJ. Strawberry gums: a sign of Wegener's granulomatosis. JAMA 1981;246(26):10-11.

6. Hoffman GS, Kerr GS, Leavitt RY. et al. Wegener granulomatosis: an analysis of 158 patients. Ann Intern Med 1992;116(6):488-98.

7. Raustia AM, Autio-Harmainen HI K, MLE RJ. U ltrastructural findings and clinical follow-up of 'strawberry gums' in Wegener's granulomatosis. J Oral Pathol 1985;14:581-7.

8. Nolle B, Specks U, Ludemann J, Rohrbach MS D, RA GW. Anticytoplasmatic autoantibodies: their immunodiagnostic value in Wegener granulomatosis. Ann Intern Med 1989;111:28-40.

9. Kundu BK GA. Gum hypertrophy - A manifestation. JIACM 2011;12:76-9.

10. CA. L. Wegener's granulomatosis: Current and upcoming therapies. Arthritis Res Ther 2003;5:180-91.

11. Ledford DK EL. Im $m$ unologic aspects of cardiovascular disease. JAMA 1987;(258):74-82.

How to cite this article: Jacob JE, Rajasekharan A, Bose TC, Ramesh S, Vinimol C, Chaurasia A. Granulomatosis with polyangitis (WEGENER'S)- Strawberry gingivitis as prime manifestation. J Oral Med, Oral Surg, Oral Pathol, Oral Radiol 2019;5(3):96-9. 